\title{
Factores determinantes en la inestabilidad del sector agrícola colombiano
}

\author{
Determining factors in Colombian agricultural sector instability
}

Recibido: 23/07/14 - Aprobado versión final: 10/10/14

\author{
Nelson E. Castaño Giraldo* \\ María A. Cardona Gómez**
}

\begin{abstract}
Resumen: este artículo expone los factores que inciden en la inestabilidad del sector agrícola colombiano y para su desarrollo se realizó una recopilación de análisis elaborados por diferentes entidades y expertos, sobre las dificultades y perspectivas que presenta el sector, y en ellos se discuten los factores más importantes que afectan su productividad y estabilidad. Los principales resultados se expresan en la influencia que tienen los conflictos sociales en la utilización del suelo, los altos costos de producción, la baja competitividad del sector y el abandono de la población rural.
\end{abstract}

Palabras clave: sector agrícola colombiano, sostenibilidad del agro, competitividad, financiación del agro en Colombia.

\begin{abstract}
Colombian agricultural sector instability. For its development, analyses on the sector's challenges and perspectives, performed by various agencies and experts, were compiled. They discuss the most significant factors affecting productivity and stability. The main findings have to do with the burden of social conflicts in the use of land, high costs of production, low sector competitiveness, and neglect rural population endures.
\end{abstract}

Keywords: colombian agricultural sector, agricultural sector's sustainability, competitiveness, agricultural funding.

JEL: Q14

* Matemático, Msc en Matemáticas Aplicadas, docente de tiempo completo adscrito a la Facultad de Ciencias Administrativas y Económicas del Tecnológico de Antioquia e integrante del Grupo de Investigación Observatorio Público de la misma institución, Medellín - Colombia. ncastano@tdea.edu.co

** Tecnóloga en Gestión Comercial e integrante del Semillero de Investigación del Grupo de Investigación Observatorio Público del Tecnológico de Antioquia, Medellín - Colombia.

ma_alejac@hotmail.es 


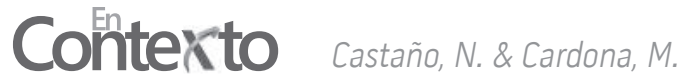

\section{Causes de l'instabilité du secteur agricole en Colombie}

Résumé: cet article présente les causes déterminant l'instabilité du secteur agricole en Colombie. Nous avons effectué une compilation des analyses réalisées par de différents organismes et experts concernant les difficultés et l'avenir du secteur. Dans ces dernières il est question des principales causes affectant la productivité et la stabilité du secteur agricole. Les principales conclusions montrent l'influence des conflits sociaux à l'égard de l'exploitation des terres, les coûts de production élevés, la faible compétitivité du secteur et l'abandon de la part de l'État de la population rurale.

Mots clé: secteur agricole en Colombie, viabilité du secteur agricole, compétitivité, financement du secteur agricole.

\section{Introducción}

La actividad agropecuaria constituye un rubro a considerar en la economía de algunos países que presentan las condiciones adecuadas para dicha actividad. Colombia es uno de los países con mayor potencial de expansión de tierras para uso agrícola en el mundo, con más de 14 millones de hectáreas (Proexport Colombia, 2013). Sin embargo la participación de este sector en el Producto Interno Bruto - PIB - ha venido decreciendo en los últimos años, pasado de un 8\% en el 2000 a un 6\% en 2013 (ver Tabla 1). La actividad cafetera, la agroindustria azucarera, los productores de cacao, los ganaderos y lecheros así como los arroceros, sustentan sus dificultades en la alta competitividad que lleva a la caída gradual de los precios internacionales, los altos costos de producción, el impacto del aumento de las importaciones, la incertidumbre frente a factores productivos como el orden público, el desplazamiento de campesinos y la legalización y restitución de tierras. 
Tabla 1. Aportes del sector agrícola al producto interno bruto nacional

\begin{tabular}{cccc}
\hline \hline Año & $\begin{array}{c}\text { Variación \% PIB } \\
\text { Nacional }\end{array}$ & $\begin{array}{c}\text { Variación \% PIB } \\
\text { Sector }\end{array}$ & $\begin{array}{c}\text { Aporte \% Sector al PIB } \\
\text { Nacional }\end{array}$ \\
\hline 2000 & 1,63 & $-2,09$ & 7,9 \\
2001 & 1,68 & 1,77 & 8,0 \\
2002 & 2,50 & 4,55 & 8,1 \\
2003 & 3,92 & 3,09 & 8,0 \\
2004 & 5,33 & 2,98 & 7,9 \\
2005 & 4,71 & 2,81 & 7,7 \\
2006 & 6,70 & 2,37 & 7,4 \\
2007 & 6,90 & 3,91 & 7,2 \\
2008 & 3,55 & $-0,38$ & 6,9 \\
2009 & 1,65 & $-0,65$ & 6,8 \\
2010 & 3,97 & 0,19 & 6,5 \\
2011 & 6,59 & 2,07 & 6,3 \\
2012 & 4,05 & 2,47 & 6,2 \\
2013 & 4,30 & 5,2 & 6,2 \\
\hline
\end{tabular}

Fuente: elaboración propia con base en datos Dane y Banco de la República.

Los altos índices de pobreza afectan considerablemente el desarrollo y la productividad del campo, en Colombia al 2013 el $46 \%$ de la población rural se encuentra en pobreza y el $21,8 \%$ en pobreza extrema (DANE, 2014), un factor que limita las posibilidades de que el crecimiento económico de la agricultura pueda distribuirse de una manera más equitativa en la población campesina, imposibilita que se mejoren las condiciones de vida en las zonas rurales y ocasiona problemáticas sociales y de orden público.

Por otro lado, en cuanto a infraestructura de transporte, es precaria la que existe, falta financiación para los nuevos proyectos, y es insuficiente la regulación y normatividad asociadas al transporte de carga, infraestructura y logística en las áreas rurales del país. Preocupa, además, el rezago en infraestructura para el control, manejo y aprovechamiento de aguas, lo que dificulta el desarrollo socioeconómico de las comunidades rurales y atenta contra la competitividad de sus emprendimientos productivos.

El gobierno viene subsanando las diferentes dificultades mediante el pago de subsidios y la implementación de reformas agrarias en las dos últimas décadas. Igualmente, ha buscado recursos para que el sector cuente con un presupuesto de inversión, pero si no se tiene claro cuáles son los criterios 


\section{Contexto castaño, N.\& \&ardona, M.}

y escenarios para la asignación eficiente de recursos públicos dentro del sector agropecuario se continuará con soluciones transitorias que no atacan la raíz del problema.

La identificación de los reales factores que inciden en la inestabilidad del sector proporcionará elementos de juicio para definir políticas públicas que aumenten los niveles de inversión en bienes públicos como infraestructura básica y servicios sociales prioritarios, y de este modo hacer atractiva y eficiente la inversión privada, para generar más y mejores empleos, posibilitar la competitividad y el sostenimiento del sector y lograr condiciones de vida digna para la población rural.

\section{Metodología}

Este documento expone una recopilación de los análisis que han hecho diferentes entidades y expertos sobre las dificultades y perspectivas que presenta el sector. En ellos se discuten los principales factores que estarían incidiendo en la baja productividad e inestabilidad reflejadas en las continuas protestas sociales y las condiciones sociales de los pequeños agricultores. Se estableció en estos análisis que la utilización de suelo influenciado por los conflictos sociales, junto con los altos costos de producción y la baja competitividad del sector, son factores relevantes, a lo que se suma el abandono de la población rural, entre la que se presentan niveles de pobreza significativos. Tales análisis se exprondrán en este artículo a partir de los hallazgos realizados al utilizar el modelo de análisis multicriterio.

\section{Resultados}

\section{Estado actual de algunas actividades agrícolas en Colombia}

El sector agrícola colombiano crece sistemáticamente por debajo de la economía y de los demás sectores. Existen diversos factores que directa o indirectamente afectan los diversos sistemas de producción de las actividades agrícolas representativas de Colombia. La actividad cafetera, por ejemplo, retrocedió de una producción de 11 millones de sacos en el 2008 a 7,3 millones en el 2012, mientras las exportaciones se desplomaron a 7,1 millones de sacos (Federacion Nacional de Cafeteros, 2014). La variabilidad de los precios internacionales más el impacto de la revaluación han incidido considerablemente en los ingresos de los exportadores, y por ende en los caficultores, lo cual se refleja en el balance comercial (ver Tabla 2).

El sector azucarero presentó dificultades por fenómenos climáticos y la competitividad con países vecinos, como lo expone el reportaje "Conozca los problemas que tienen en jaque al agro en Colombia": 
Debido a fenómenos climáticos, la molienda de caña en los ingenios descendió 16,4\% entre enero y junio del 2013, al llegar a 8,7 millones de toneladas, contra 10,4 millones en igual lapso del 2012. A lo anterior se agrega el impacto de las crecientes importaciones del granulado, especialmente de Brasil (54\%) y de Bolivia (24\%), las cuales entre enero y mayo del 2013 alcanzaron las 171.000 toneladas. Esa competencia ha provocado una pérdida de competitividad por tasa de cambio del 18,3\%, ya que mientras Brasil devaluó su moneda (el real) en 9,9\% en 2012, Colombia revaluó el peso en 8,4\% en ese año. (El País, 2013)

Los alto costos de los agroquímicos en los últimos tres años han afectado a los cacaoteros, sin embargo, el Gobierno ha otorgado subsidios por $\$ 105.000$ millones como una forma de estimular las siembras y los precios internos deprimidos por las importaciones, y \$9.000 millones para estabilización de precios. (El País, 2013)

Los arroceros se enfrentan hoy a bajos precios y altos costos de producción. En el 2013 los precios internos descendieron entre un $10 \%$ y un $20 \%$, esto debido a las crecientes importaciones desde Estados Unidos, de donde han entrado 65.000 toneladas y se prevé la llegada de 17.500 toneladas más. Además, se espera que ingrese más arroz extranjero por cuenta de los nuevos tratados comerciales, en especial desde la Alianza del Pacífico. (El País, 2013)

Según la balanza comercial del sector agropecuario y agroindustrial, sin el aporte de la producción cafetera, viene acarreando dese el 2010 un déficit en sus ingresos debido a los bajos precios en el mercado internacional y el alto costo de las importaciones (Tabla 2). 


\section{Contexto castaño, N.\& \&ardona, M.}

Tabla 2. Balanza comercial del sector agrícola colombiano

\begin{tabular}{|c|c|c|c|}
\hline \multicolumn{4}{|c|}{$\begin{array}{l}\text { Balanza comercial del sector agropecuario y agroindustrial } \\
\text { (Miles de dólares) }\end{array}$} \\
\hline Año & $\begin{array}{l}\text { Total agropecuaria y } \\
\text { agroindustrial sin café }\end{array}$ & Café & $\begin{array}{l}\text { Total agropecuaria y } \\
\text { agroindustrial con café }\end{array}$ \\
\hline 2000 & $\$ 475.046$ & $\$ 1.067 .761$ & $\$ 1.542 .807$ \\
\hline 2001 & $\$ 442.508$ & $\$ 760.090$ & $\$ 1.202 .598$ \\
\hline 2002 & $\$ 441.489$ & $\$ 767.469$ & $\$ 1.208 .958$ \\
\hline 2003 & $\$ 433.893$ & $\$ 803.717$ & $\$ 1.237 .610$ \\
\hline 2004 & $\$ 613.064$ & $\$ 958.731$ & $\$ 1.571 .795$ \\
\hline 2005 & $\$ 1.117 .374$ & $\$ 1.467 .444$ & $\$ 2.584 .819$ \\
\hline 2006 & $\$ 970.939$ & $\$ 1.433 .754$ & $\$ 2.404 .693$ \\
\hline 2007 & $\$ 869.226$ & $\$ 1.721 .392$ & $\$ 2.590 .618$ \\
\hline 2008 & $\$ 518.012$ & $\$ 1.896 .929$ & $\$ 2.414 .941$ \\
\hline 2009 & $\$ 886.554$ & $\$ 1.490 .038$ & $\$ 2.376 .593$ \\
\hline 2010 & $-\$ 300.766$ & $\$ 1.834 .208$ & $\$ 1.533 .441$ \\
\hline 2011 & $-\$ 851.038$ & $\$ 2.452 .017$ & $\$ 1.600 .979$ \\
\hline 2012 & $-\$ 1.315 .734$ & $\$ 1.780 .513$ & $\$ 464.779$ \\
\hline
\end{tabular}

Fuente: elaboración propia con base en de datos Dane, SAC

y Departamento de Estudios Agroeconómicos.

Todos estos factores y algunos otros demandan del Estado la preocupación y la atención para el sector, a fin de establecer planes y políticas eficientes que permitan determinar a dónde deben apuntar los recursos financieros para hacerlo competitivo y atractivo para la inversión. El Gobierno nacional debe orientar sus esfuerzos a fortalecer la institucionalidad pública, asignar mayores recursos en materia de financiamiento y desarrollar una estrategia de inclusión social.

\section{Dificultades en la productividad del sector agrícola colombiano}

Como toda actividad económica, la producción agrícola se compone de diversos elementos, cada uno de ellos esencial y con funciones específicas dentro de la cadena de producción. La tierra, la mano de obra, las técnicas de producción y la infraestructura componen la primera fase, en la cual se estiman los costos de producción y se establecen los recursos necesarios. Luego, en la segunda fase, se comercializa la producción con un valor que compita con los precios del mercado interno e internacionales. Para la sostenibilidad se requiere, por lo menos, el equilibrio económico entre los costos de producción y los precios de comercialización, buscando la rentabilidad de la actividad económica. El desequilibro en la balanza en contra de la actividad agrícola se da por los altos costos de producción y la baja competitividad de la misma. 
Hay un cúmulo de razones que explican el pobre desempeño del sector agrícola colombiano. Se destacan los problemas de orden público y la alta sensibilidad del sector a la consabida precariedad de la infraestructura de transporte en el país. Fedesarrollo ha señalado que el mal desempeño del sector agrícola tiene que ver, además de los elementos recién mencionados, con la política comercial aplicable al agro, la cual históricamente ha sido altamente proteccionista.

En la actualidad, Colombia presenta dificultades en las bases primarias de la producción reflejadas en la baja productividad y los altos costos de producción, lo que genera limitaciones de competitividad, algunas de las razones son: la deficiente disponibilidad de infraestructura y de logística; las barreras que enfrenta la población rural para desarrollar su potencial productivo, por la falta de competencias laborales; el tema de tierras, la calidad de vida; las técnicas y tecnologías utilizadas en el proceso de producción, y las dificultades que desestimulan la inversión. A continuación se desarrollan los principales elementos que estarían en el origen de la baja competitividad del sector.

\section{Tierras}

\section{Sobrecosto de fertilizantes}

Las condiciones del suelo en Colombia no son favorables para la producción competitiva del sector agrícola, los suelos de alta calidad ocupan una pequeña porción del territorio nacional, lo que obliga a la utilización de fertilizantes en zonas ácidas del país. Colombia es uno de los países del mundo que más cantidad de fertilizantes usa por hectárea cultivada. De acuerdo con datos del Banco Mundial, entre el 2009 y 2013 en el país se usaron 575.3 toneladas de fertilizantes por hectárea cultivable (Banco Mundial, 2014), por lo cual se hace relevante el análisis de su valor y su incidencia en los costos de producción.

Los precios de los fertilizantes representan un porcentaje significativo en los costos de producción de productos transitorios y permanentes. Para la producción de arroz, algodón, papa y maíz blanco, por ejemplo, los precios de los fertilizantes representan entre el 20 y el $30 \%$ de sus costos. Por el lado del café, el cacao, la palma y la caña panelera, van desde el 15 hasta el 30\%. (Marín, 2013)

Los productos básicos para la elaboración de fertilizantes son importados, en el artículo "Los dueños de los fertilizantes en Colombia” (Marín, 2013), se argumenta que: 


\section{Contexto Castaño, N. \& cardona, M.}

En el año 2012 ingresaron al país: 516.000 toneladas de úrea, 422.000 toneladas de potasio y 70.000 toneladas de fosfato. Además de las mezclas de estos productos, que alcanzaron las 342.000 toneladas, en total llegaron al país casi 1,4 millones de toneladas de fertilizantes básicos, a un costo (incluyendo flete) de casi US\$708 millones, es decir, casi \$1,4 billones.

La importación de estos insumos hace que el precio lo definan los distribuidores y empresas importadoras. Por la falta de regulación del Gobierno estos productos están propensos a sobrecosto en el mercado interno con respecto a los precios internacionales (ver Figura 1).

Al comparar los precios internacionales con los nacionales, se observa un sobrecosto entre el 30 y el $50 \%$ por un kilo de fertilizantes, esto debido a que las seis empresas que dominan el mercado definen el precio interno. En diciembre de 2012 un kilo de urea se conseguía fuera de las fronteras en $\$ 897$ mientras las sociedades comercializadoras lo vendieron en Colombia a \$1.248 para un sobrecosto del $40 \%$. Para el caso del potasio, el precio promedio internacional fue de $\$ 764$, el interno llegó a los \$1.223 por kilo, una diferencia del 60\%. El fosfato se pagó a \$681 en el exterior y en el país a \$1.489. El sobrecosto alcanzó el 118\%. (Marín, 2013)

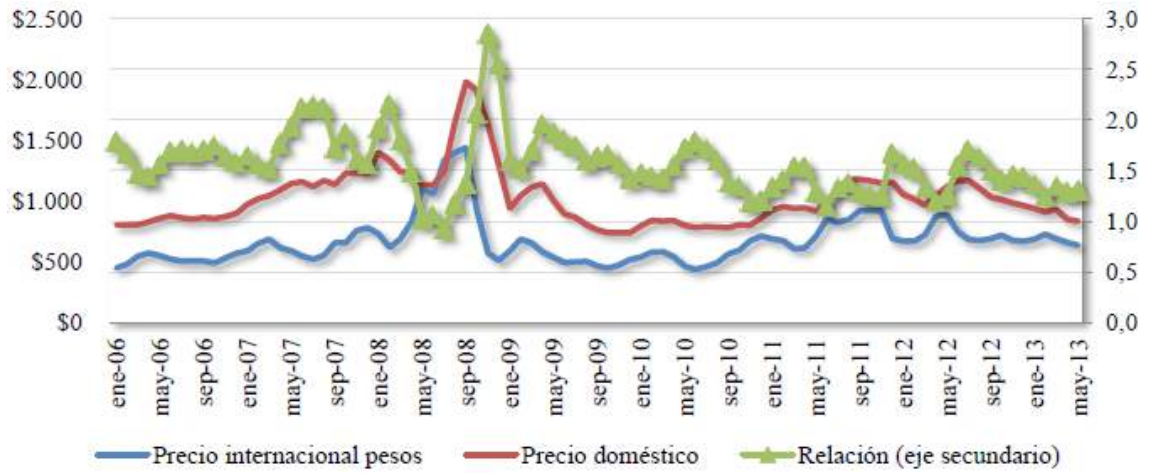

Figura 1. Comparativo precios internacionales y precios domésticos úrea (enero de 2006 a abril de 2013)

Fuente: cálculos GEE-SIC a partir de Agronet y MADR (2013b).

Desde 2006 el Ministerio de Agricultura monitorea los precios de los agroinsumos, pero no interviene para corregir. El precio de los fertilizantes en Colombia representa un factor determinante para la pérdida de rentabilidad y competitividad en la agricultura colombiana. 


\section{Uso de tierras}

Colombia es uno de los países con mayor potencial de expansión de tierras para uso agrícola en el mundo. Según la FAO, Colombia se ubica en el puesto 25 entre 223 países en la evaluación del potencial de expansión del área agrícola sin afectar el área de bosque natural (SAC, 2012). Este potencial de crecimiento, según el Ministerio de Agricultura y Desarrollo Rural, se estima en 10 millones de hectáreas, dentro de las que se encuentran áreas no aprovechadas y otras que tradicionalmente se han utilizado sin atender criterios de vocación productiva.

A partir del diagnóstico y las recomendaciones de política para mejorar la competitividad del sector agropecuario colombiano, se afirma:

El potencial de uso de las tierras en Colombia es limitado, aunque ese no es el problema mayor. El principal problema es la mala asignación de esas tierras. Por una parte, el potencial de tierras para agricultura es de 10,4 millones de hectáreas pero el uso actual llega a 4,2 millones de hectáreas. En tierras de vocación agroforestal hay un potencial de 21,9 millones de has, pero el uso actual es de 10 millones. En tierras de uso exclusivo forestal el potencial es de 21,6 millones de has y su uso actual es de 9,9 millones. En cambio, en ganadería el potencial es de 10,2 millones de has y su uso actual es de 41,7 millones. Las tierras de conservación tienen un potencial de 49,6 millones de has y un uso de 47,9 millones de has. El exceso de tierra destinada a la ganadería (alrededor de 30 millones de has) a costa de la agricultura (defecto de 6 millones de has) y el sector forestal (defecto de 24 millones de has) representa uno de los principales retos a modificar para posibilitar un salto en el crecimiento del sector. (Consejo Privado de Competitividad, 2008)

Las grandes extensiones de tierra utilizadas para ganadería de baja productividad, las restricciones legislativas sobre tenencia de la tierra en Colombia y la posesión de tierras de vocación agrícola en poder de personas al margen de la ley o en manos del Estado por extinción de dominio, están impidiendo un crecimiento dinámico del sector en cuanto a tierras se refiere.

Es prioritario que las autoridades adelanten la extinción de dominio sobre las tierras que aún permanecen en poder de estas personas al margen de la ley y, dado que la vocación del Estado no es ser empresario agrícola, lo que debería suceder de manera pronta es que se les restituya las tierras a la población que las perdió $y$ desee retornar o las venda o alquile a los empresarios que las quieren explotar. (Consejo Privado de Competitividad, 2008)

El acceso de la población rural a tierras de buena calidad es muy limitado, tierras planas, con agua y con infraestructura son utilizadas en una ganadería poco tecnificada y poco productiva. Por otro lado, la problemática social debido al vínculo entre el conflicto, el desplazamiento y la concentración 


\section{Contexto castaño, N.\& \&ardona, M.}

de la propiedad territorial agudizan el problema. Entre 1980 y julio de 2010, 434.100 familias desplazadas se vieron obligadas a abandonar, vender o entregar a terceros forzosamente 6.638.195 hectáreas (Perry, 2010). El desplazamiento forzado ha llevado a una mayor concentración de la propiedad territorial en Colombia y a incrementar dramáticamente la población desposeída en el país y en sus zonas ruarles.

\section{Desarrollo rural y calidad de vida}

En Colombia la pobreza se concentra desproporcionadamente en el campo. Colombia tiene $45 \%$ de pobreza, pero en el sector rural es de $64 \%$ (Perry, 2010) y esto se ha visto agravado por el conflicto armado, el desplazamiento, el despojo de tierra y los cultivos ilícitos, y dificulta la capacidad de generar inversión pública y privada en el campo colombiano.

En las áreas rurales colombianas viven 11.838 .032 personas, el $26 \%$ de la población nacional. El $62,1 \%$ de ellos, es decir 7.351 .418 personas, viven en la pobreza, y el 21,5\% de la población rural (2.545.177 personas) viven en pobreza extrema o indigencia (Perry, 2010). De manera que cerca de las dos terceras partes de los moradores rurales son pobres y más de la tercera parte de los pobres rurales se haya en la indigencia.

A pesar de los relativos avances que en crecimiento y modernización se han presentado en el agro colombiano, los niveles de pobreza y de miseria han disminuido muy lentamente. No sobra mencionar que la mayoría de la población rural en Colombia depende directamente del sector agropecuario para su subsistencia, y que la mayoría de ella tiene ingresos muy bajos: el $70 \%$ de los ocupados en el sector agropecuario devenga menos de un salario mínimo. Este porcentaje en el conjunto del sector rural es de $68 \%$, mientras que en el urbano este porcentaje es menos de la mitad (31\%). (Leibovich, 2006)

Factores estructurales, como el bajo acceso a activos productivos (tierras, recursos hídricos y financiamiento) en cantidades adecuadas para desarrollar sistemas productivos eficientes, limitan la generación de ingresos suficientes y sostenibles en las zonas rurales. Adicionalmente, el despojo de tierras y la presencia de los cultivos ilícitos, junto con la ausencia de políticas sociales en cuanto a la seguridad social, educación y servicios públicos deterioran gravemente la calidad de vida en estas zonas.

\section{Infraestructura}

Una de las dificultades que atenta contra la competitividad y los emprendimientos productivos del sector es la precaria infraestructura vial, principal medio de transporte de los productos agropecuarios, y el poco desarrollo de otros medios como el marítimo y el férreo. Según Santiago Perry: 
Colombia cuenta con cerca de $162.000 \mathrm{~km}$ de vías que conforman el total de la red vial nacional. La red primaria - que está a cargo de la Nación - está conformada por algo más de $16.000 \mathrm{~km}$, la red secundaria (a cargo de los departamentos) por $71.000 \mathrm{~km}$, y la red terciaria por cerca de $60.000 \mathrm{~km}$. Si bien en todas ellas hay serias deficiencias de mantenimiento, la falta de recursos de la mayoría de los entes territoriales del país hace que la situación sea especialmente crítica en la secundaria y en la terciaria, precisamente las más importantes para la salida de los productos de las fincas campesinas a los centros de acopio y consumo. (2010)

El abandono de la red vial terciaria en regiones lejanas o de difícil acceso dificulta realizar un proceso de comercialización con las especificaciones que exigen los mercados formales e institucionales, lo que deja a los pequeños productores en manos de los intermediarios que controlan los mercados informales y los someten a vender sus productos en condiciones desfavorables situación que genera sobrecostos significativos en la comercialización de los bienes agrícolas.

Otro factor a tener en cuenta es la deficiente infraestructura para el control, manejo y aprovechamiento de aguas. El potencial de tierras irrigables en Colombia es de 7 millones de hectáreas aproximadamente, y sólo cerca de 900 mil hectáreas disponen de infraestructura para riego o drenaje. De ellas, el sector público ha adecuado cerca de una cuarta parte, a costos significativamente elevados y con una muy baja recuperación de las inversiones públicas, además de las problemáticas administrativas de mantenimiento y operatividad que se generan. Para completar, las investigaciones y las realizaciones en estrategias económicas e innovadoras para dotar de agua para riego a comunidades rurales pobres son incipientes y poco difundidas en Colombia. La carencia de agua determina que los pequeños productores pierdan con frecuencia sus cosechas o vean seriamente disminuidos sus rendimientos, imposibilitando diversificar su producción hacia cultivos que les generen mayores ingresos (frutas, hortalizas, entre otros.).

El preocupante rezago en infraestructura dificulta el desarrollo socioeconómico de las comunidades que las habitan y atenta contra la competitividad de sus emprendimientos productivos. Según la Encuesta de Opinión Empresarial Agropecuaria - EOEA -:

La proporción de productores que afirmaron haber realizado inversiones en maquinaria y equipo, instalaciones e infraestructura y adecuación de tierras fue menor que en 2012. Las mayores caídas se registraron en el rubro de inversión en construcción de instalaciones y obras de infraestructura, pues el porcentaje de productores agropecuarios que afirmó hacerlas cayó 7,1 puntos porcentuales, pasando de $22,9 \%$ a $15,7 \%$, seguido. (SAC, 2013) 


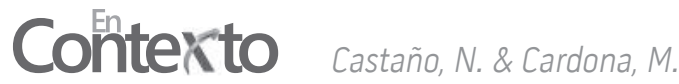

\section{Innovación tecnológica}

Con el fin de mejorar la competitividad del sector a través de mayores rendimientos, altos niveles de calidad y posicionamiento en los mercados, es necesario consolidar la incorporación de nuevas técnicas, métodos e insumos, que aumenten la eficiencia y la productividad de los sistemas productivos y la comercialización de los productos. Por esa razón, dentro del direccionamiento estratégico del sector, la variable tecnológica toma relevancia.

De acuerdo con el Departamento Nacional de Planeación (2007), el incremento de la productividad agropecuaria y el desarrollo de sistemas productivos altamente competitivos requieren del uso eficiente de los factores productivos primarios y, fundamentalmente, del desarrollo de procesos de innovación tecnológica que mejoren el uso de estos factores, y permitan incrementar los rendimientos de la producción. Es allí donde la ciencia y la tecnología se convierten en elementos diferenciadores en el crecimiento económico del sector agropecuario, reconocimiento que el Ministerio de Agricultura y Desarrollo Rural - MADR - ha hecho al mencionar que la empresarización de la agricultura exige inversión en ciencia y tecnología, para lograr incrementar los rendimientos, reducir costos y, por esta vía, poder competir con otros países. (Ministerio de Agricultura y Desarrollo Rural, 2010)

En cuanto a competitividad, productividad e innovación, un análisis de brechas en los diferentes sectores realizado en el Departamento Nacional de Planeación encontró que en Colombia nadie está encargado de los temas de transferencia de tecnología en el sector agropecuario. Obviamente, el Instituto Colombiano Agropecuario - ICA - y la Corporación Colombiana de Investigación Agropecuaria - Corpoica - hacen investigación, pero no se encargan de la transferencia de tecnología. El Centro Internacional de Agricultura Tropical - CIAT - también está haciendo estudios, pero no hay quién esté dedicado, en forma sistemática a este tema, lo que pone en evidencia un vacío inmenso.

La investigación y la promoción deben centrarse en fortalecer las ventajas competitivas, en vez de persistir en políticas centradas en subsidios que no han reducido la pobreza rural. (Botero, 2012)

\section{Inversión y financiamiento}

El financiamiento del sector agropecuario en Colombia ha sido restringido y segmentado (Banco Mundial, 2014). El sector está sujeto no sólo a los riesgos de clima y plagas sino a la volatilidad de los precios que, en el caso de los bienes transables, dependen de los precios internacionales y la tasa de cambio, variables no controlables. La historia de refinanciaciones y condonaciones masivas de deudores agropecuarios, en especial durante la década de los 
noventa, por efecto de los bajos precios internacionales y la revaluación del peso, produjo un estigma sobre el sector que generó una aversión mayor del sistema financiero a prestarle. En efecto, en 2003 sólo el 8\% de las empresas rurales tenían acceso a crédito formal, estando concentrado en aquellos segmentos de la producción agropecuaria integrada con la agroindustria. Desde el punto de vista del sector financiero, es más atractivo focalizar su negocio en las áreas urbanas del país. Las garantías exigidas para los créditos formales en la actividad agropecuaria son más difíciles de cumplir, y en general los costos de transacción son más elevados en las áreas rurales.

En la medida en que haya un segmento cada vez más empresarial de la producción agropecuaria, existen posibilidades crecientes de que la banca privada encuentre atractivo prestarle. Es más difícil que la pequeña producción sea sujeta de crédito por parte de la banca privada, pues las normas de límite a la tasa de interés a través de la tasa de usura hacen difícil que los costos de transacción en créditos a pequeños productores se puedan recuperar. Por esta razón, tiene sentido que la política agropecuaria haya privilegiado recursos públicos para otorgar financiamiento al sector.

Sin embargo, esta política se ha hecho a través de inversiones forzosas del sistema financiero, lo que le resta competitividad al sector. De otra parte, como los recursos de crédito vía Finagro (Fondo para el Financiamiento del Sector Agropecuario) son limitados, ha habido racionamiento y en su mayoría han sido destinados a medianos y grandes productores que, en muchos casos, podrían tener acceso al sistema financiero tradicional, y así liberar recursos para ser asignados a pequeños productores.

\section{La posición del Estado ante las dificultades del sector agrícola en Colombia}

Las acciones orientadas a la población pobre rural se han concentrado en programas de Acción Social que no cuentan con una adecuada coordinación con la política agrícola ni tienen necesariamente como fin generar en los pobres rurales las capacidades productivas para que puedan convertirse en pequeños empresarios rurales en el mediano plazo, y liderar sus propios procesos de desarrollo. Los programas del Ministerio de Agricultura difícilmente llegan a los pobladores pobres del campo, a pesar de su intencionalidad.

En cuanto a la posesión de la tierra, la manera de inducir un cambio en esta situación es a través del cobro del impuesto predial sobre tierras improductivas con base en una tasa razonable y un avalúo actualizado. Así, los propietarios se verán incentivados a poner a producir las tierras o en su defecto a venderlas. El Gobierno nacional y el Congreso de la República aprobaron la Ley 1152 de 2007 de Desarrollo Rural que contiene los artículos 72 y 73 por los cuales el Estado queda autorizado para comprar tierras improductivas al valor del avalúo catastral del año anterior. En caso de que el propietario se resista a efectuar la transacción, el Estado podrá expropiar la tierra previa 


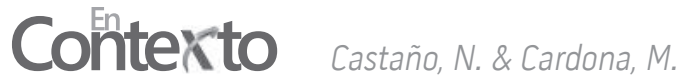

indemnización que será equivalente al avalúo catastral del año anterior. Aún falta que el Gobierno nacional reglamente la Ley y empiece a actuar. Por el simple efecto de demostración del Estado se podría generar un proceso de cambio en el uso de las tierras en la dirección deseable.

En el 2012 se crearon tres nuevas entidades que entrarán a complementar y fortalecer el trabajo del Ministerio de Agricultura y Desarrollo Rural y las entidades ya existentes. En uso de las facultades extraordinarias, otorgadas mediante la Ley 1444 de 2011, se crearon las unidades encargadas de la restitución de tierras: Unidad Administrativa Especial de Gestión de la Restitución de Tierras Despojadas; la planificación de las tierras rurales y su uso agropecuario: Unidad de Planificación de Tierras Rurales, Adecuación de Tierras y Usos Agropecuarios, y el manejo integral y aprovechamiento sostenible de los recursos pesqueros: Autoridad Nacional de Acuicultura y Pesca.

En lo referente a la restitución, la Unidad Administrativa Especial de Gestión de Restitución de Tierras Despojadas, que tiene a su cargo la materialización de la restitución de tierras a personas afectadas por el despojo y el abandono forzoso, cuenta hasta el momento con trece oficinas en diferentes ciudades del país, que han recibido más de 14.000 solicitudes correspondientes a 1.110.626 hectáreas que fueron despojadas.

Igualmente, el Ministerio de Agricultura y Desarrollo Rural viene trabajando arduamente para atacar el problema de la informalidad en la tenencia de la tierra que se estima en cerca del $40 \%$. Con este fin, se ha creado en la entidad el programa de Formalización de la Propiedad Rural que ha venido promoviendo procesos administrativos y judiciales para brindar seguridad jurídica a poseedores y tenedores informales de predios. Para llevar a cabo el proceso, se han identificado unas Zonas de Formalización Masiva, ubicadas en once departamentos, en las que se hará un barrido predial masivo para sanear la propiedad de estas tierras.

El fortalecimiento presupuestal del Ministerio de Agricultura y Desarrollo Rural y sus entidades permitirá llegar a más productores con instrumentos para el mejoramiento competitivo.

- Instrumentos para incrementar la producción y la productividad: crédito agropecuario, Incentivo a la Capitalización Rural - ICR -, Fondo Agropecuario de Garantías - FAG -, adecuación de tierras, investigación y desarrollo tecnológico, asistencia técnica agropecuaria, sanidad agrícola y pecuaria, Conpes lácteo.

- Programas para ampliar y diversificar el mercado interno y externo: Plan País Maíz y Plan Decenal de Desarrollo Cacaotero.

- Gestión del riesgo agropecuario: seguro agropecuario, coberturas de tasa de cambio y de precio internacional, Plan Nacional de Reforestación y Sistemas de Información del Sector Agropecuario. 
Además, el Gobierno viene implementando una variedad de instrumentos de política dispuestos para apoyar a los productores agropecuarios en coyunturas difíciles y aumentar su competitividad, entre otros el programa de coberturas cambiarias, el seguro agropecuario y el programa Desarrollo Rural con Equidad (DRE) para el fomento a la competitividad. Se busca introducir la normatividad que debe regir para el logro real del desarrollo de las zonas rurales del país, y a su vez organizar la dispersa legislación existente sobre reforma agraria, en un cuerpo único y coherente que facilite su aplicación y consulta. Esto también permitirá la consolidación de los procesos de restitución de tierras que se vienen adelantando en el marco de la Ley 1448 de 2011, y estimular su inserción a las actividades agropecuarias.

En los últimos años, los gobiernos han tomado conciencia de la importancia del manejo del riesgo en la agricultura como herramienta esencial para anticipar, evitar o reaccionar ante choques externos. Varios países de la Organización para la Cooperación y el Desarrollo Económicos - OCDE - han desarrollado sistemas de gestión del riesgo para evitar el deterioro de las condiciones de vida de aquellos que dependen de la agricultura para su sustento. En Colombia, los sistemas de gestión del riesgo son aún incipientes y poco desarrollados. Sin embargo, en los últimos años el MADR ha hecho un esfuerzo por poner en marcha algunos instrumentos que permitan a los productores agropecuarios mitigar los efectos de fenómenos externos y reducir su vulnerabilidad. Existen en la actualidad dos tipos de herramientas para la gestión del riesgo: el programa de coberturas y el seguro agropecuario.

\section{Conclusiones}

Son numerosos los factores que inciden en la situación actual del agro colombiano. La pobreza rural continúa siendo un problema que repercute directamente en la productividad y competitividad del sector agrícola y la acción gubernamental en la materia no ha tenido un significativo impacto para reducirla. Los avances obtenidos han sido escasos, el acceso de la población rural a servicios sociales, públicos, educativos y de seguridad social es aún precario. La carencia de una infraestructura adecuada de aguas, así como su control, manejo y aprovechamiento dificultan el desarrollo socioeconómico de las comunidades que habitan las zonas rurales, y atenta contra la competitividad de sus emprendimientos productivos. Además, la dificultad de acceso a la tierra y a otros factores productivos agudiza la situación, la falta de estrategias y de políticas apropiadas para apoyar los esfuerzos de desarrollo socioeconómico y de generación de ingresos de las comunidades rurales pobres han proporcionado el estancamiento del sector.

Se requieren estrategias más apropiadas para acompañar a la población rural pobre en sus procesos de desarrollo económico. Las entidades del Estado, en ocasiones, no han encontrado su papel para apoyar los procesos de desarrollo de los pobres rurales ni a las organizaciones ya constituidas de 


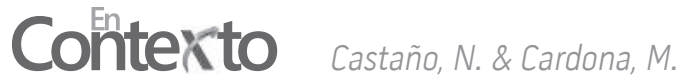

pequeños productores rurales, para el fortalecimiento de su producción y para la comercialización conjunta de las cosechas.

El Estado ha subsanado en las dos últimas décadas las diferentes dificultades mediante el pago de subsidios y con la implementación de reformas agrarias, pero se deben establecer políticas públicas que proporcionen mayores garantías de sostenibilidad del sector y de inversión en él, y que los entes estatales y privados lo incluyan en las líneas de crédito.

Se han descrito e identificado los factores que pueden estar incidiendo en el estancamiento y la inestabilidad del sector agrícola, y se proporcionan elementos de juicio para establecer la necesidad de políticas públicas que aumenten los niveles de inversión en bienes públicos, como infraestructura básica y servicios sociales prioritarios, que propicien el desarrollo integral del campo y su entorno rural, haciéndolo productivo y competitivo, de modo que la inversión privada se haga atractiva y eficiente, se generen más y mejores empleos, y se logren condiciones de vida digna para sus pobladores; única vía posible para aprovechar competitiva y sosteniblemente las amplias oportunidades de desarrollo que ofrece el sector.

\section{Referencias bibliográficas}

Banco Mundial. (2014). Datos. Consumo de fertilizantes (kilogramos por hectárea de tierras cultivables). Recuperado de http://datos.bancomundial. org/indicador/AG.CON.FERT.ZS/countries?display=default

Botero, J. (2012, 10 de abril). Retos y desafíos del agro. Portafolio. Recuperado de http://www.portafolio.co/opinion/retos-y-desafios-del-agro

Consejo Privado de Competitividad. (2008). Competitividad del sector agropecuario colombiano. Recuperado de http://www.compite.com. co/site/wp-content/uploads/informes/2008-2009/Agropecuario(agricultura).pdf

Colombia, DANE (2013, 31 de julio). Pobreza Monetaria Año Móvil Julio 2012-Junio 2013. Boletín de prensa. Recuperado de https://www.dane.gov. co/files/investigaciones/condiciones_vida/pobreza/bol_pobreza_jun13.pdf

Colombia, Departamento Nacional de Planeación. (2007). Aprovechar las potencialidades del campo.

Marín, A. (2013, 7 de septiembre). Los dueños de los fertilizantes en Colombia. El Espectador. Recuperado de http://www.elespectador.com/noticias/ investigacion/los-duenos-de-los-fertilizantes-colombia-articulo-445007 
El País. (2013, 11 de agosto). Conozca los problemas que tienen en jaque al agro en Colombia. Recuperado de El País. http://www.elpais.com.co/elpais/ economia/noticias/estos-son-problemas-tienen-jaque-agro-colombia

Federacion Nacional de Cafeteros. (2014). Estadísticas históricas. Información estadística cafetera. Recuperado de http://www.federaciondecafeteros.org/ particulares/es/quienes_somos/119_estadisticas_historicas/

Leibovich. J., Nigrinis, M. \& Ramos, M. (2006). Caracterización del mercado laboral rural en Colombia. Recuperado de http://www.banrep.gov.co/ docum/ftp/borra408.pdf

Colombia, Ministerio de Agricultura y Desarrollo Rural. (2010). Memorias 2006-2007. Recuperado de http://www.minagricultura.gov.com

Perry, S. (2010). La pobreza rural en Colombia. Centro Latinoamericano para el Desarrollo Rural - RIMISP — Recuperado de http://www.rimisp.org/wpcontent/files_mf/1366386291DocumentoDiagnosticoColombia.pdf

Proexport Colombia. (2013, 30 de septiembre). Sector agroindustrial de Colombia muestra su potencial de proveeduría en Alemania durante Anuga 2013. Recuperado de http://www.proexport.com.co/noticias/ sector-agroindustrial-de-colombia-muestra-su-potencial-de-proveeduriaen-alemania-en-anuga-2013

SAC. (2013). Los precios de venta se imponen como la mayor preocupación de los productores. Encuesta de opinión Empresarial Agropecuaria. Boletín de prensa. Sociedad Agrícola Colombiana. Recuperado de http://www. sac.org.co/images/eoea/2013/JUL-SEP_ETAPA_56/COMUNICADO_ DE_PRENSA_ETAPA_57.pdf

\section{Para citar este artículo:}

Castaño, N. \& Cardona, M. (2014). Factores determinantes en la inestabilidad del sector agrícola colombiano. En-Contexto, 2, 91-107 